(C) [2008] IEEE. Reprinted, with permission, from Wang, Shuhong; Liu, Xinying; Qiu, Jie; Zhu, Jianguo; Guo, Youguang; Lin, Zhi Wei (Jack). 2008, Robust Optimization in HTS Cable Based on DEPSO and Design for Six Sigma', Proceedings of the 43rd IEEE Industry Applications Society Annual Meeting, pp. 1-5.This material is posted here with permission of the IEEE. Such permission of the IEEE does not in any way imply IEEE endorsement of any of the University of Technology, Sydney's products or services. Internal or personal use of this material is permitted. However, permission to reprint/republish this material for advertising or promotional purposes or for creating new collective works for resale or redistribution must be obtained from the IEEE by writing to pubs-permissions@ieee.org. By choosing to view this document, you agree to all provisions of the copyright laws protecting it. 


\section{Robust Optimization in HTS Cable Based on DEPSO and Design for Six Sigma}

\author{
Shuhong Wang, Xinying Liu, Jie Qiu \\ State Key Laboratory of Electrical Insulation and Power \\ Equipment, Faculty of Electrical Engineering \\ Xi’an Jiaotong University, Xi’an, 710049 China \\ shwang@mail.xjtu.edu.cn
}

\author{
Jian Guo Zhu, Youguang Guo and Zhi Wei Lin \\ Faculty of Engineering \\ University of Technology, Sydney \\ Sydney, P.O. Box 123, NSW 2007, Australia \\ joe@eng.uts.edu.au
}

\begin{abstract}
The non-uniform AC current distribution among the multi-layer conductors in a high-temperature superconducting (HTS) cable reduces the current capacity and increases the AC loss. In this paper, Particle swarm optimization coupled with differential evolution operator (DEPSO) has been applied in structural optimization of HTS cables. While the existence of fluctuation in design variables or operation conditions has a great influence on the cable quality, in order to eliminate the effects of parameter perturbations in design and improve the design efficiency, a robust design method based on design for six sigma (DFSS) is applied in this paper. The optimization solutions show that the proposed optimization procedure can not only achieve a uniform current distribution, but also improve significantly the reliability and robustness of the HTS cable quality.
\end{abstract}

Keywords-current distribution; high temperature superconducting (HTS) cable; particle swarm optimization (PSO); perturbation analysis; differential evolution (DE) operator; design of six sigma.

\section{INTRODUCTION}

HTS cables for large current transmission in general have a multi-layer structure consisting of parallel connected tapes, twisted in each layer. Due to the difference of inductances among layers, the currents flowing in these layers are different. Therefore, the control of current distribution among these layers is an important issue for design and optimization of an HTS cable because this significantly affects the current transmission capacity and power losses.

Usually, for an HTS AC cable, the current distribution among layers is substantially determined by the inductive impedances of these layers. The distribution of inductive impedances is however dependent on the structural parameters of the cable conductor. The main method to obtain a uniform current distribution is to alternate the inductive impedances of layers by adjusting the structural parameters of the cable conductors. Many optimization methods, such as the genetic algorithm (GA) and the particle swarm optimization (PSO) algorithm, have been applied [1]. In this paper, an improved PSO with differential evolution operator (DEPSO) is presented to optimize the structural parameters of HTS cable conductors to achieve the uniform current distribution among layers.

However, the performance of an HTS cable may to a certain degree be affected by perturbation of parameters possibly caused by imperfect manufacturing or non-ideal properties of superconducting tapes, e.g. shrinkage at lowtemperature [2]. Traditional optimization methods cannot take into account the perturbations, so they may lead to unreliable or non-robust solutions.

In this paper, a robust design method based on design for six sigma (DFSS) [3] is introduced for HTS cable optimization. The philosophy of DFSS in quality engineering is applied in this optimization procedure to improve the process quality and design reliability. Taking a cold dielectric type HTS cable as an example, the optimized parameters are compared with those obtained by DEPSO.

\section{IMPROVED PARTICLE SWARM OPTIMIZATION METHOD}

\section{A. Particle Swarm Optimization (PSO)}

The particle swarm optimization (PSO) method is a population based stochastic optimization technique developed in 1995 by Kennedy and Eberhart, inspired by the social behavior of birds flocking and fish schooling [4].

Suppose that the search space has D-dimensions. The position of the $i$-th particle in the swarm can then be expressed as a vector $X_{i}(t)=\left(X_{i, 1}(t), X_{i, 2}(t), \ldots, X_{i, D}(t)\right)$. The velocity of this particle can be represented by another vector $V_{i}(t)=\left(V_{i, 1}(t)\right.$, $\left.V_{i, 2}(t), \ldots, V_{i, D}(t)\right)$. The $i$-th particle also maintains a memory of its previous best position in the vector pbest $t_{i}$, and in each iteration step, gbest is designated as the index of the best particle in the swarm. Subsequently, the swarm is manipulated according to the following two equations [5]:

$$
\begin{aligned}
V_{i, d}(t)= & w V_{i, d}(t-1)+c_{1} r_{1} \times\left(\text { pbest }_{i, d}-X_{i, d}(t-1)\right) / \Delta t \\
& +c_{2} r_{2} \times\left(\text { gbest }_{d}-X_{i, d}(t-1)\right) / \Delta t
\end{aligned}
$$

and

$$
X_{i, d}(t)=X_{i, d}(t-1)+V_{i, d}(t) \cdot \Delta t
$$

where $d=1,2, \ldots, D$, and $i=1,2, \ldots, N, N$ is the size of the swarm, $c_{1}$ and $c_{2}$ are two positive constants, namely social and cognitive parameters, $r_{1}$ and $r_{2}$ two random numbers distributed within the range $[0,1], t$ is the iteration number, $\Delta t=1$, and $w$ is inertia weight. 


\section{B. DEPSO Algorithm}

To overcome the premature of multi-model function search by the standard PSO, a hybrid particle swarm with differential evolution operator (DEPSO) is utilized, which also provides the bell-shaped mutations with consensus on the population diversity, while keeps the particle swarm dynamics [6].

The mutations are provided by DE operator on the pbest ${ }_{i}$, with a trail point tbest $_{i}=$ pbest $_{i}$, which for the $d$ th dimension:

$$
\begin{aligned}
& \text { If }(\operatorname{rand}()<\text { CR OR } d==k) \\
& \text { tbest }_{i, d}=\text { pbest }_{i, d}+\lambda \cdot\left(\text { gbest }_{d}-\text { pbest }_{i, d}\right)+\beta \cdot \delta_{2, d} \\
& \delta \Delta, 2=\left(\begin{array}{ll}
\sum_{1}^{2} & d
\end{array}\right) / \\
& \Delta p=\text { best }_{A, R}-\text { best }_{B, d}
\end{aligned}
$$

where $k$ is a random integer value within $[1, D]$, which ensures the mutation at least one dimension, $C R$ is a crossover constant, $\lambda$ and $\beta$ are two weighted factors respectively, $\delta_{2}$ is the general difference vector, $\Delta$ means the difference between two elements that are randomly chosen from a common point set, which includes all the pbest in the current case, pbest $_{A}$ and pbest $_{B}$ are chosen from the pbest set at random.

\section{RobUSt OptimizATION USING DESIGN FOR SiX SigMA}

Because of errors and uncertainties in design process, manufacturing process, and operating condition in real-world engineering designs, the idea of robust optimization considering both the optimality and the robustness of objective function and constraints has been paid attention for real-world design problems in recent years.

Design for six sigma (DFSS) is one of conventional robust optimization approaches. The term "sigma” refers to standard deviation $\sigma$, which is a measure of dispersion, and "six sigma" is one of the management reform techniques aiming at the establishment of business process with very small dispersion.

In a traditional optimization (minimization) problem, the objective function $f$ of design variable $\boldsymbol{X}$ should be minimized as follows.

\section{Minimize: $f(\boldsymbol{X})$}

Combining the probabilistic elements of reliability, robust design, and six sigma, variability is incorporated into a robust optimization formulation through the definition of uncertain random variables, formulation of reliable input constraints and output constraints, and objective robustness (minimize variation in addition to mean performance on target). The formulations of robust optimization based on DFSS can be established as follows [3]:

$$
\begin{array}{ll}
\text { Minimize: } & F\left(\mu_{f}(X), \sigma_{f}(X)\right) \\
= & \mu_{f}(X)+\omega_{1} \sigma_{f}(X) \\
\text { subject to: } & \mu_{g_{i}(X)}-n \sigma_{g_{i}(X)} \geq 0 \\
& X_{L}+n \sigma_{X} \leq \mu_{X} \leq X_{U}-n \sigma_{X} \\
& \mu_{f}-n \sigma_{f} \geq \text { Lower specification limit } \\
& \mu_{f}+n \sigma_{f} \leq \text { Upper specification limit }
\end{array}
$$

where the set of design variables $\boldsymbol{X}$ includes the input parameters that may be design variables, random variables, or both. The objective function $F$ and constraints are described by the mean value $\mu_{f}(\boldsymbol{X})$ and standard deviation $\sigma_{f}(\boldsymbol{X})$.

The main steps to achieve DFSS are described as Fig. 1 [7].

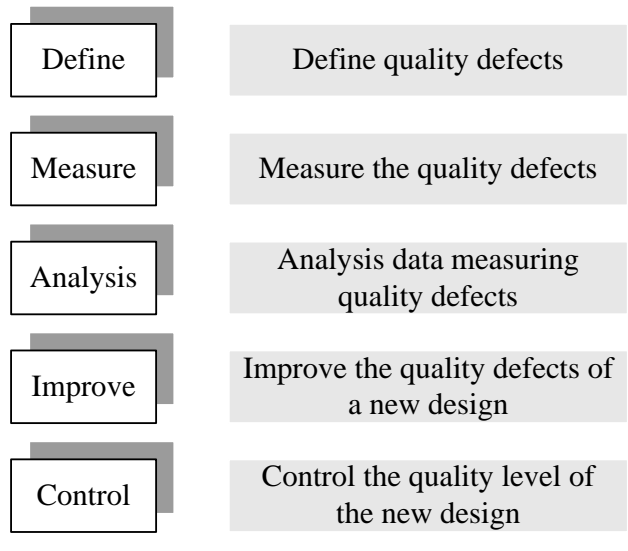

Figure 1. DFSS procedure

\section{MODEl OF HTS CABle}

The structure of single-phase cold dielectric type HTS power cable, consisting of four layers of conductors and two layers of shield of $\mathrm{Ag} / \mathrm{Bi}-2223$ tapes, is shown in Fig. 2 [8].

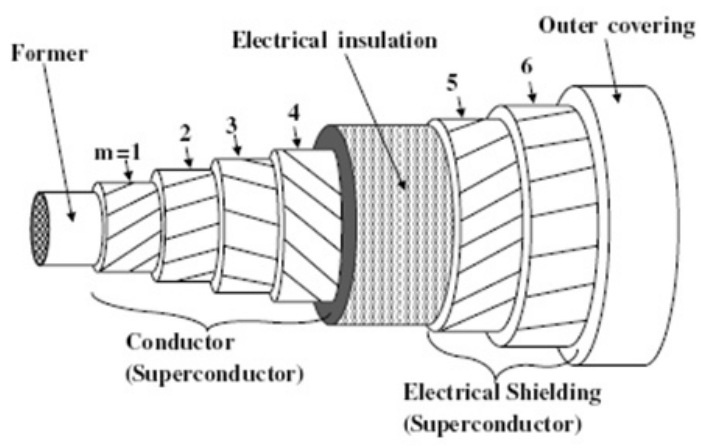

Figure 2. Schematic diagram of the cold dielectric type HTS cable.

As described in [1], the winding angle, direction and radius of each layer are selected as the design variables. For a cable of $n$ layers, the optimized variables can be expressed as a vector:

$$
\boldsymbol{X}=\left[\beta_{1}, a_{1}, R_{1}, \beta_{2}, a_{2}, R_{2}, \cdots, \beta_{n}, a_{n}, R_{n}\right]
$$


Without quenching, the objective function for optimization of the cold dielectric type HTS cable is derived to achieve the uniform current distribution among the phase conductors and the shields respectively, which is subject to the mechanical properties and critical current of the tape. The critical current is derived from its relationship of the magnetic field and temperature of superconducting tape.

$$
\begin{array}{r}
\min f(\boldsymbol{X})=\sum_{i=1}^{m-1} \sum_{j=i+1}^{m}\left|I_{i x}(\boldsymbol{X})-I_{j x}(\boldsymbol{X})\right|+\sum_{i=1}^{m-1} \sum_{j=i+1}^{m}\left|I_{i y}(\boldsymbol{X})-I_{j y}(\boldsymbol{X})\right| \\
+\sum_{i=m+1}^{n-1} \sum_{j=i+1}^{n}\left|I_{i x}(\boldsymbol{X})-I_{j x}(\boldsymbol{X})\right|+\sum_{i=m+1}^{n-1} \sum_{j=i+1}^{n}\left|I_{i y}(\boldsymbol{X})-I_{j y}(\boldsymbol{X})\right|
\end{array}
$$

where $I_{i x}(\boldsymbol{X})$ and $\mathrm{I}_{i y}(\boldsymbol{X})$ are the real and imaginary components of current $\dot{I}_{i}(\boldsymbol{X})$ in the $i$-th layer. $\dot{I}_{i}(\boldsymbol{X})$ as a function of the parameter vector $\boldsymbol{X}$ can be derived from [1]. The current distribution among layers should become more uniform when $f(X)$ is closer to a minimal value.

The associated constraints are:

\section{1) Constraints of mechanical properties}

Considering the mechanical properties of a tape, such as the tensile strain characteristic and the bending strain characteristic, the constraints of mechanical properties can be expressed as

$$
\left\{\begin{array}{l}
\beta_{i}-\arcsin \left(\frac{2 R_{i} \varepsilon_{c b}}{t}\right)^{1 / 2} \leq 0 \\
\beta_{i}-\arcsin \left(\frac{\varepsilon_{c t}+\varepsilon_{p}-\varepsilon_{f c}}{\varepsilon_{p}-\varepsilon_{r}}\right)^{1 / 2} \geq 0
\end{array} \quad i=1,2, \cdots, n\right.
$$

where $\varepsilon_{c b}$ and $\varepsilon_{c t}$ are the critical bending and tensile strain of the tape at $77 \mathrm{~K}, \varepsilon_{p}$ and $\varepsilon_{f c}$ the thermal shrinkages of the winding pitch and the tape, respectively, $\varepsilon_{r}$ is the radial thermal shrinkage of the former, and $t$ the thickness of the tape.

\section{2) Constraints of radii}

The constraints of radii can be expressed as

$$
\left\{\begin{array}{l}
R_{1}-\frac{D_{\min }}{2}+\left(t_{f}+\frac{t}{2}\right) \geq 0 \\
R_{i+1}-R_{i}-\left(t_{f}+t\right) \geq 0 \quad i=1,2, \cdots, n-1 \\
\frac{D_{\max }}{2}-R_{i}-(n-i)\left(t_{f}+t\right)-\frac{t}{2} \geq 0 \quad i=1,2, \cdots n
\end{array}\right.
$$

where $D_{\min }$ and $D_{\max }$ are used to limit the inner and outer diameters of the cable conductors, and $t_{f}$ is the thickness of the dielectric between layers.

\section{3) Constraints of critical current}

These constraints are used to restrict the currents in layers below their critical currents, and can be expressed as

$$
I_{i}<N_{i} I_{c} k_{1} k_{2} k_{3} k_{4} \quad i=1,2, \cdots, n
$$

where $N_{i}$ is the number of tapes wound on the $i$-th layer, $I_{c}$ the mean of critical currents of HTS tapes in the cable, $k_{1}, k_{2}$ and $k_{3}$ are the deteriorations of the critical current considering the magnetic field and the temperature, manufacture, and the thermal cycles, respectively, and $k_{4}$ is the design safety margin.

\section{STRUCTURAL PARAMETER OPTIMIZATION}

Taking a cold dielectric type HTS cable with 4-layer conductors and 2-layer shields as an example, Table I tabulates the structural parameters of the cold dielectric type HTS cable before the structural optimization.

TABLE I. Structural PARAMETERS OF COLD Dielectric TyPe

\begin{tabular}{|c|c|c|c|c|c|c|}
\hline LAYER INDEX & 1 & 2 & 3 & 4 & 5 & 6 \\
\hline$\alpha_{i}$ & +1 & +1 & -1 & -1 & +1 & +1 \\
\hline$\left.\beta_{i}{ }^{\circ}\right)$ & 27.0 & 27.0 & 27.0 & 27.0 & 27.0 & 27.0 \\
\hline$R_{i}(\mathrm{~mm})$ & 10.0 & 10.45 & 10.90 & 11.35 & 18.50 & 18.95 \\
\hline
\end{tabular}

Note: $\alpha$ is the winding direction, $\beta$ the winding angle, $R$ the radius; Layers 1-4 are conductors and Layers 5 and 6 the shields.

The length of the cable for calculation is chosen to be 100 $\mathrm{m}$. The AC voltage source $u$ is $10 \mathrm{kV}$ (rms), and the load $R_{L}$ is $10 \Omega$. Fig. 3 plots the current distributions before the structural optimization. It is found that the currents in different layers before optimization differ greatly in both the amplitude and phase angle.

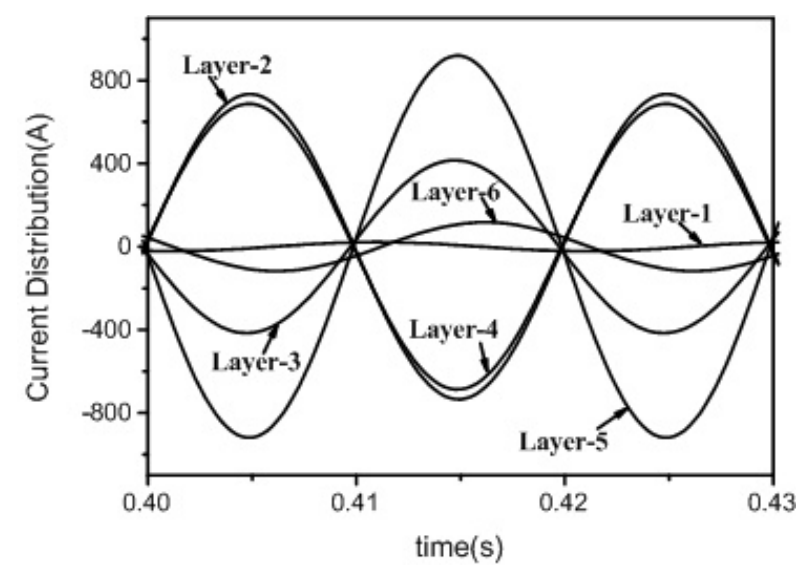

Figure 3. Current distribution in the cold dielectric type HTS cable before optimization

\section{1) Comparision between PSO and DEPSO}

PSO and DEPSO are performed under the same condition. A uniform current distribution, shown in Fig. 4, could be achieved with both two algorithms, and the comparison between PSO and DEPSO is listed in Table II, and also illustrated in Fig. 5. In Table II, $F_{\text {best }}$ is the average of the best fitness function value of 50 evolutions, and $T_{\text {iter }}$ is the average of the iterative times of 50 evolutions. It can be concluded that DEPSO could have more opportunities to find out the optimal result and could converge easily. In Fig. $5, F_{\text {norm }}$ is defined as 
the average of the best fitness function value of 50 evolutions in the same generation. The result shows that DEPSO provides a higher performance than PSO when the number of generations is greater than 1200 .

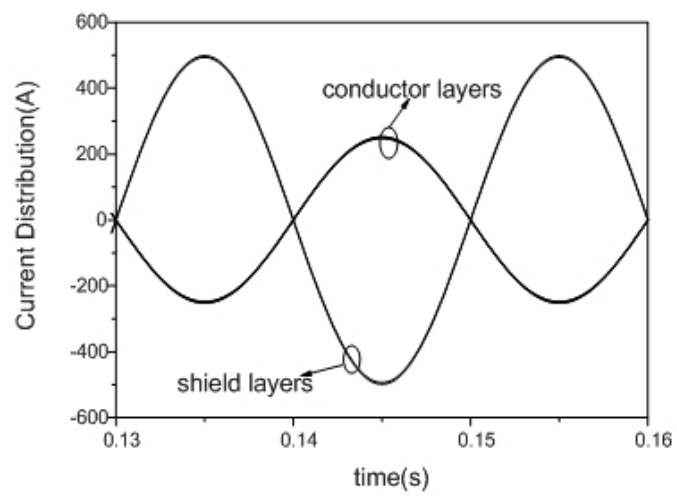

Figure 4. Current distribution in the cold dielectric type HTS cable after optimization

TABLE II. PERFORMANCE COMPARISION BETWEEN PSO AND DEPSO

\begin{tabular}{|c|c|c|}
\hline Algorithm & $F_{\text {best }}$ & $T_{\text {iter }}$ \\
\hline PSO & 1039.07 & 4948.48 \\
\hline DEPSO & 244.786 & 3189.14 \\
\hline
\end{tabular}

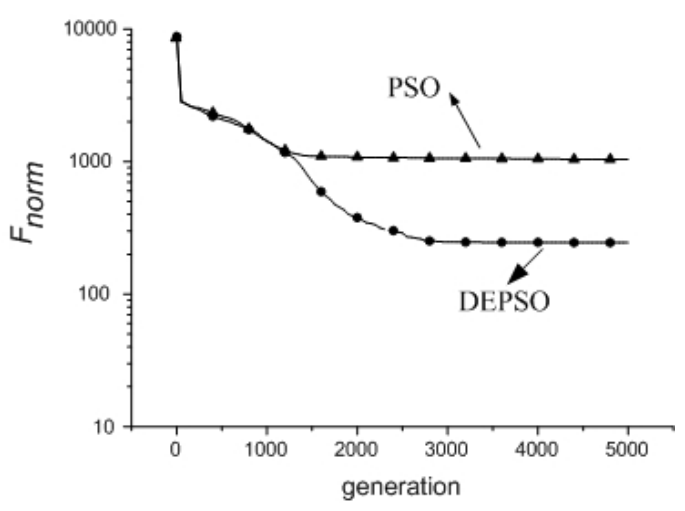

Figure 5. Performance of PSO and DEPSO

\section{2) Comparision between DEPSO and DFSS}

When the perturbation range of design variables is $\pm 0.1 \%$, the DEPSO algorithm and robust optimization with DFSS are performed. And the comparisons of the optimized structural parameters are shown in Table III.

From Table III, it can be seen that the current distributions optimized by DEPSO and DFSS are almost the same. Fig. 6 demonstrates the probability distribution of fitness function $F$ in different optimization results. It can be seen that in DEPSO the distribution covers a wider range, while in DFSS the probability distribution shrinks much thinner. By this way, the DFSS optimization increases the robustness of the design.

Table IV shows the quality improvement by using DFSS. With DEPSO algorithm, the mean value of the objective function $\mu_{F}=101.729$, the standard deviation is $\sigma_{F}=56.677$ and the reliability is $63.8723 \%$. By using the six sigma robust optimization, the mean value and the standard deviation of the objective function decreased to 54.52 and 24.67, respectively. The constraints have almost $0 \%$ probability in exceeding their limits.

TABLE III. OPTIMIZED STRUCtURAL PARAMETERS OF HTS CABLE

\begin{tabular}{|c|c|c|c|c|c|c|}
\hline \multirow{2}{*}{$\begin{array}{c}\text { Layer } \\
\text { Index }\end{array}$} & \multicolumn{3}{|c|}{ DEPSO Algorithm } & \multicolumn{3}{|c|}{ DFSS Optimization } \\
\cline { 2 - 7 } & $a_{i}^{\Delta}$ & $\beta_{i}^{\Delta}$ & $R_{i}^{\Delta}(\mathrm{mm})$ & $a_{i}^{*}$ & $\beta_{i}^{*}$ & $R_{i}^{*}(\mathrm{~mm})$ \\
\hline 1 & -1 & 16.23 & 9.69 & -1 & 24.15 & 9.00 \\
\hline 2 & -1 & 8.03 & 10.11 & -1 & 8.00 & 9.91 \\
\hline 3 & 1 & 8.92 & 10.64 & 1 & 10.37 & 10.69 \\
\hline 4 & 1 & 30.64 & 10.99 & 1 & 30.00 & 11.55 \\
\hline 5 & -1 & 21.94 & 17.94 & -1 & 22.81 & 18.05 \\
\hline 6 & -1 & 10.59 & 18.51 & -1 & 14.41 & 19.21 \\
\hline
\end{tabular}

Note: $\Delta$ represents PSO algorithm, and * DFSS optimization; the definitions of $\alpha, \beta$ or $R$ is similar to those in Table I.

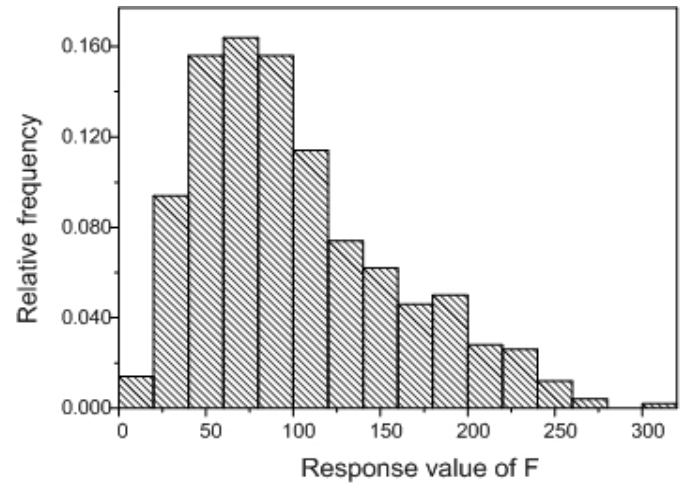

a) DEPSO algorithm

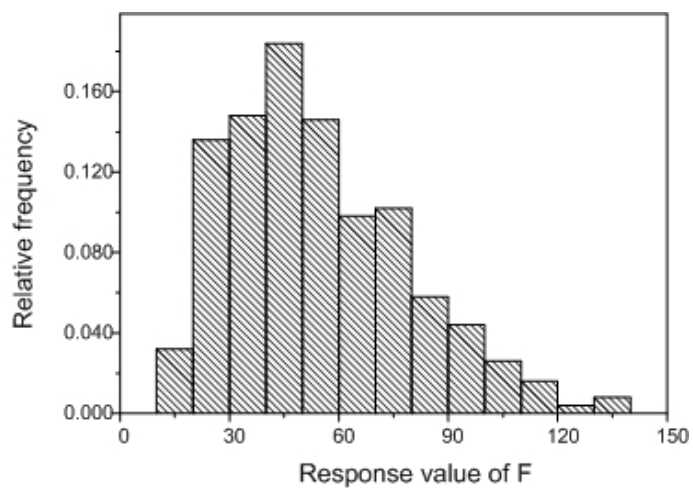

b) Robust optimization based on DFSS

Figure 6. Histogram of DEPSO algorithm and robust design for six sigma

TABLE IV. QUALITY IMPROVEMENT FOR HTS CABLE

\begin{tabular}{|c|c|c|c|}
\hline Optimization & $\mu_{F}$ & $\sigma_{F}$ & Reliability \\
\hline DEPSO & 101.729 & 56.677 & $63.8723 \%$ \\
\hline DFSS & 54.5188 & 24.6709 & $\sim 100 \%$ \\
\hline
\end{tabular}


The perturbation analysis is applied to evaluate the influence of the distorted structural parameters. The current relative error of the $i$-th layer is introduced to investigate the current distribution with perturbed parameters as follows

$$
E_{c v, i}=\frac{\left|I_{a v e}-I_{e r r, i}\right|}{I_{a v e}} \times 100 \%
$$

where $I_{\text {ave }}$ is the average value of the currents of the layers obtained through the optimized parameters, and $I_{e r r, i}$ the current of the $i$-th layer with the perturbed structural parameters.

The perturbations are performed on the winding angle and the radius in a certain range. The maximum value of $E_{\mathrm{cv}}$ in all cases is defined as $E_{\max }$. The curves in Fig. 7 reveal that the robust stabilization of DFSS is higher than that of PSO.
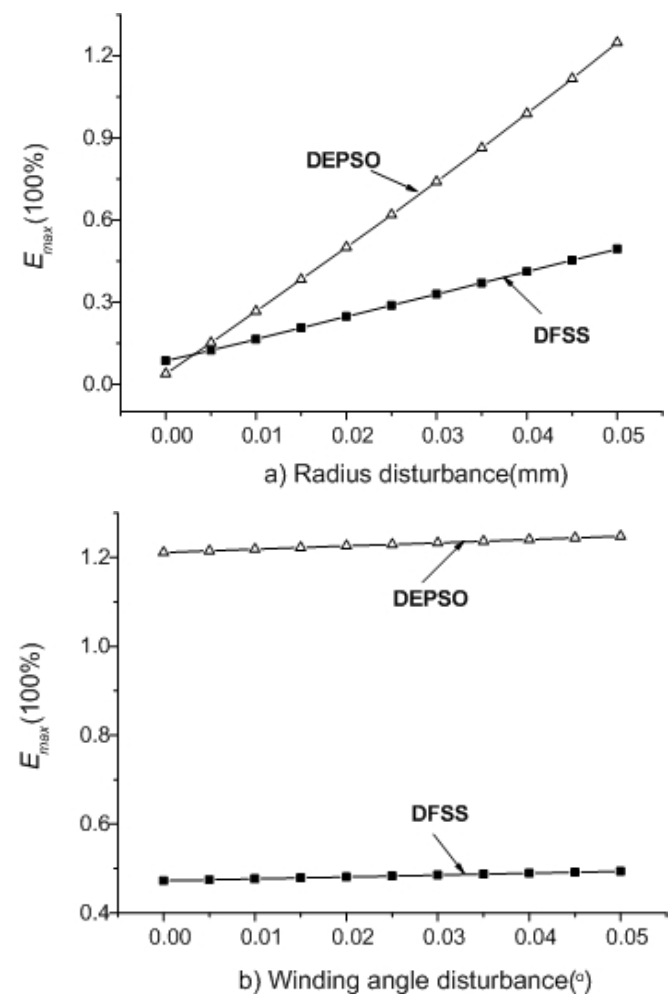

Figure 7. Influence of parameters perturbation on current distribution

\section{CONCLUSION}

In this paper, an improved particle swarm optimization, which is hybrid traditional PSO with differential evolution operator, is utilized to overcome the premature of multi-model function search by the standard PSO, and it has proved that DEPSO provides a better performance than PSO. Considering the uncertainties in HTS cable structural design, a optimization algorithm based on design of six sigma is applied to perform a robust design. The comparison between DEPSO and robust optimization shows that the robust optimization using design of six sigma is superior to the DEPSO algorithm to achieve higher reliability and quality.

\section{REFERENCES}

[1] Shuhong Wang, Jie Qiu, Zhen Zhao, et al., "Robust optimization of multilayer conductors of HTS AC cable using PSO and perturbation analysis," in Proc. 41 ${ }^{\text {st }}$ IEEE Industry Applications Society Annual Meeting, , Oct. 2006, pp. 293-299.

[2] M. Tsuda, A. K. M. Alamgir, Y. Ito, et al., "Homogeneous current distribution in a coaxial superconductor with and without return current path,” IEEE Trans. Appl. Supercond., vol. 11, no. 1, pp. 481-484, 2001.

[3] P. N. Koch, R. J. Yang, and L. Gu, "Design for six sigma through robust optimization,” Struct. Multidisc. Optim., vol. 26, pp. 235-248, 2004.

[4] R. Eberhart and J. Kennedy, "A new optimizer using particle swarm theory," in Proc. $6^{\text {th }}$ International Symposium on Micro Machine and Human Science, Japan, 1995, pp. 39-43.

[5] Y. Shi and R. Eberhart, “A modified particle swarm optimizer,” in Proc. IEEE International Conference on Evolutionary Computation, USA, 1998, pp. 69-73.

[6] Zhang Wenjun and Xie Xiaofeng, "Hybrid particle swarm with differential evolution operator,” in Proc. IEEE International Conference on Systems, Man and Cybernetics, 2003, pp. 3816-3821.

[7] H. S. Kim, C. B. Kim, and H. J. Yim, "Quality improvement for brake judder using design for six sigma with response surface method and sigma based robust design," International Journal of Automotive Technology, vol. 4, no. 4, pp. 193-201, 2003.

[8] H. Noji, K. Haji, and T. Hamada, "Alternating current loss calculation in a high-Tc superconducting transmission cable considering the magnetic field distribution,” Supercond. Sci. Technol., vol. 16, pp. 14-18, 2003. 